\title{
All-Optical Self-Synchronizing Scheme for Contention Resolution in Asynchronous Optical Packet Switched Networks Using Continuously Tunable Optical Delay Line
}

\author{
Claudio Porzi ${ }^{(1)}$, Sanghoon Chin ${ }^{(2)}$, Andrea Trita ${ }^{(3)}$, Francesco Fresi $^{(1)}$, Gianluca Berrettini ${ }^{(1)}$, Gabor Mezosi ${ }^{(4)}$, \\ Paolo Ghelfi $^{(5)}$, Guido Giuliani ${ }^{(3)}$, Luca Potì $^{(5)}$, Marc Sorel $^{(4)}$, Luc Thévenaz ${ }^{(2)}$, Antonella Bogoni ${ }^{(5)}$ \\ 1: CEIICP,Scuola Superiore Sant'Anna, Via Moruzzi 1, 56124 Pisa, Italy \\ 2: Ecole Polytechnique Fédérale de Lausanne, STI-GR-SCI-LT Station 11, 1015 Lausanne, Switzerland \\ 3: Dipartimento di Elettronica, University of Pavia, Via Ferrata 1, i-27100 Pavia, Italy \\ 4: Dept. of Electronics and Electrical Engineering, University of Glasgow, Glasgow G12 8LT, U.K. \\ 5: National Laboratory of Photonic Networks, CNIT - Via G. Moruzzi 1, 56124, Pisa, Italy \\ e-mail: claudio.porzi@sssup.it
}

\begin{abstract}
Continuously tunable, optically-controlled variable delay line based on dynamic Brillouin grating is exploited for automated synchronization of switching signals in a network node, enabling asynchronous contention resolution with packet priorization.

OCIS codes: (060.1155) All-optical networks; (060.4259) Networks, packet-switched; (130.4815)Optical switching devices
\end{abstract}

\section{Introduction}

Continuously tunable, variable optical delay lines (ODLs) can overcome the limitations of fixed optical buffers for packets synchronization in asynchronous optical packet switched networks (OPSNs). All-optical asynchronous contention resolution (CR) without fixed delay-line buffering has been recently proposed [1,2]. However, all the previous approaches exploited latching mechanisms which only enabled first-come-first-served policy, thus preventing to enforce packet priorization.

By using a variable ODL, we propose an all-optical self-synchronizing CR mechanism for asynchronous OPSNs, operating on a priority basis. In this demonstration, priority is assigned to the input port; priority can be also assigned to a specific packet by exploiting priority labels. Another main advantage of the proposed solution lies in a considerable reduction of active elements; furthermore, it is not dependent on the data bit-rate. The circuit exploits an ODL based on stimulated Brillouin scattering (SBS-ODL) [3], an integrated all-optical flip-flop based on a semiconductor ring laser (SRL-AOFF) [4], and two integrated semiconductor optical amplifier Mach-Zehnder interferometers (SOA-MZIs).

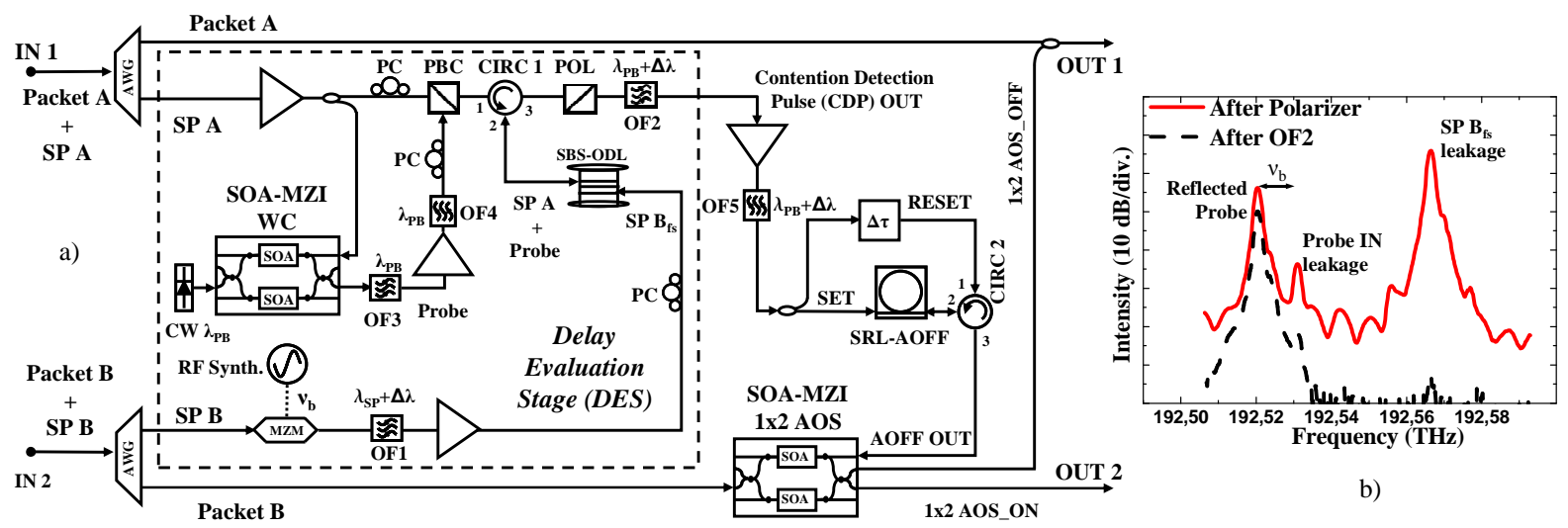

Fig. 1. a): Experimental setup. b): Output spectra at the SBS-ODL.

\section{Setup and principle}

Fig. 1a) shows the experimental set-up. Two packets, Packet A and Packet B with same wavelength $\lambda_{\text {pck }}$ and duration $\mathrm{T}_{\mathrm{pck}}$, enter the node inputs IN 1 and IN 2, respectively, at randomly different instants. IN 1 is chosen to be the high-priority (HP) port. Our architecture exploits synchronization pulses (SPs), signaling the arrival of the

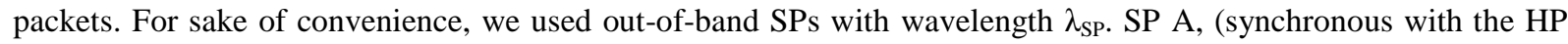
packet, Packet A), enters the node from IN 1, whereas SP B (synchronous with the LP packet, Packet B) enters the node from IN 2. Input SPs and packets are then separated using arrayed waveguide grating (AWG) filters. Packet A 


\section{JWA46.pdf}

is sent to the intended node output, OUT 1, whereas Packet B is sent to a SOA-MZI 1x2 all-optical switch (AOS). The SPs are sent to a delay evaluation stage (DES), which is the core part of the circuit, and comprises a SOA-MZI wavelength converter (WC), a Mach-Zehnder modulator (MZM)-based single-sideband wavelength converter driven by a sinusoidal tone at $10.53 \mathrm{GHz}$, and the SBS-ODL.

The SBS-ODL consists of a span of polarization-maintaining fiber (PMF), whose transmit time is equal to the length of packets, $\mathrm{T}_{\text {pck}}$. The variable SBS-ODL exploits the generation of localized Brillouin dynamic grating (BDG) using the SBS nonlinear interaction of two counter-propagating pump waves in the PMF, under the condition that the two waves are spectrally separated by the Brillouin frequency shift of the fiber, $v_{\mathrm{b}}$. In the birefringent PMF, it is possible to generate a BDG by two waves propagating along one polarization axis and observe the scattering from this Bragg-like grating for an orthogonally polarized probe pulse at a shifted frequency from the writing waves [3]. By modulating SP B at the Brillouin frequency $v_{b}=10.53 \mathrm{GHz}$ through the MZM and retaining the lower frequency sideband with an optical filter (OF 1), a frequency-shifted replica of SP B, hereafter named SP $\mathrm{B}_{\mathrm{fs}}$, is generated at $\lambda_{\mathrm{SP}}+\Delta \lambda$. SP A and SP $\mathrm{B}_{\mathrm{fs}}$ are then linearly polarized along the PMF fast axis and launched from opposite ends of the SBS-ODL. An acoustic grating is then created at the crossing point of the two pulses within the fiber. A fraction of SP A is also sent to the WC to produce a probe pulse at an optical frequency which is $45 \mathrm{GHz}$ below the frequency of SP A. The optical frequency of the probe is chosen to maximize the reflection from the BDG for a signal propagating along the slow axis of the PMF. The probe pulse is thus linearly polarized along the PMF slow axis and launched into the PMF, following SP A with a time interval of $2.5 \mathrm{~ns}$ (see Fig. 2a). A reflected probe pulse, experiencing a variable round-trip propagation depending on the relative input timing between SP A and SP $B_{\mathrm{fs}}$ (i.e., the delay between the input packets), can then be retrieved at the DES output by means of a polarizer (POL) and OF2. The DES output is then sent to the AOFF.

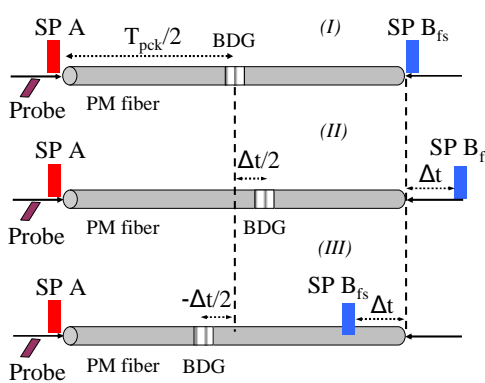

a)

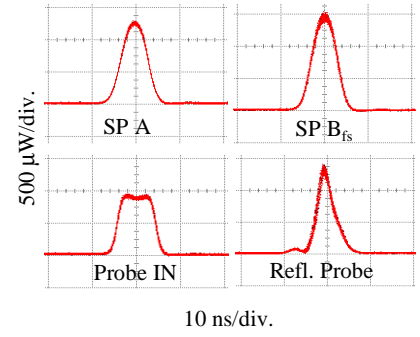

b)

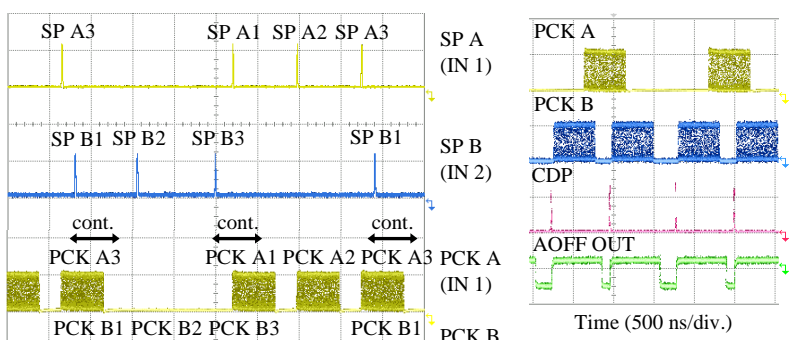

d)

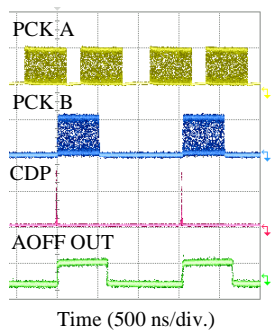

e)

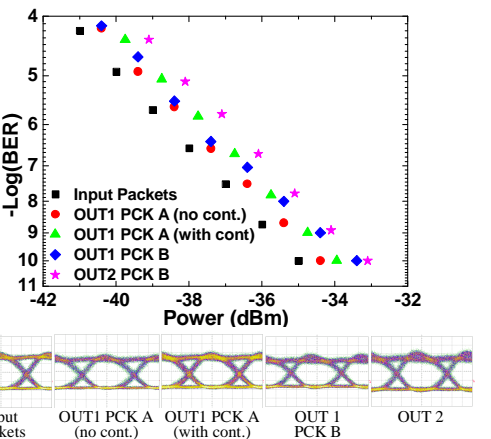

f)

Fig 2: a): Operation principle of the SBS-ODL; b): time traces of input/output signals at the DES; c), d), e): oscilloscope traces for different contention cases; f): BER measurements and corresponding eye diagrams.

The DES operation principle can then be explained as it follows: if the two packets are completely separated in time at the node inputs (case of no contention), then SP A and SP $\mathrm{B}_{\mathrm{fs}}$ do not encounter within the PMF, since its transmit time equals packets duration. No BDG is created, and the probe pulse propagates through the PMF without undergoing reflection. No signal is thus generated at the DES output. The AOFF after OF 5 remains thus idle, and no switching signal is sent to the $1 \times 2$ AOS from the AOFF output (toward OUT 1 in Fig 1). Packet B thus exits the 1x2 AOS_OFF output port and both Packet A and B are routed toward the intended node output (OUT 1), for this no contention case. If the two packets arrive synchronously at the node inputs, SP A and SP $\mathrm{B}_{\mathrm{fs}}$ enter the PMF at the same time (case of Fig. 2a-I). A BDG is created in the middle of the fiber, and a reflected probe contention detecting 
pulse (CDP) is produced at the ODL output with a total delay of $\mathrm{T}_{\text {pck }}$. The CDP is split into two pulses. One pulse is sent to the SET input port of the AOFF, and the second pulse enters the RESET input port of the AOFF with a proper delay such to produce a switching signal slightly larger than $T_{p c k}$ at the AOFF output. The path of Packet B is fixed so that Packet B is synchronous with the switching signal at the $1 \times 2$ AOS. Packet B is now deflected toward the 1x2 AOS_ON output (OUT 2 of the node in Fig. 1a) for this contention case. If Packet B enters the node delayed (advanced) with respect to Packet A by $\Delta \mathrm{t}$, being $0<\Delta \mathrm{t}<\mathrm{T}_{\mathrm{pck}}\left(-\mathrm{T}_{\mathrm{pck}}<\Delta \mathrm{t}<0\right)$, then SP A enters the fiber before (after) SP $B_{\mathrm{fs}}$ by the same amount of time (see Fig. 2a-II and 2a-III, respectively). The BDG is then created farther from (closer to) the probe input side, with a time offset of $\Delta \mathrm{t} / 2$ with respect to the middle of the fiber. The reflected probe CDP is then delayed (advanced) by $\Delta \mathrm{t}$, and the corresponding switching signal generated by the AOFF is still synchronized with Packet B at the $1 \times 2$ AOS, thus demonstrating asynchronous CR in the space domain without packet fragmentation. By inserting an extra SOA-MZI at the 1x2 AOS_ON output, and using Packet B as the pump signal and the AOFF output as the probe signal, contention can be also resolved in the wavelength domain by transferring data modulation at the AOFF signal wavelength. Fig. 1b) shows the SBS-ODL output spectra for the contention case. A strong reflected probe component (with the ordinary Doppler shift of $v_{\mathrm{b}}$ ) is evident.

\section{Results}

Fig. 2c) shows the oscilloscope traces of the signals along the circuit for the above described cases. We used $10 \mathrm{~Gb} / \mathrm{s}$ modulated optical packets with $\mathrm{T}_{\mathrm{pck}}=512 \mathrm{~ns}$ (corresponding to $\sim 100 \mathrm{~m}$ of PMF for the SBS-ODL), and $5 \mathrm{~ns}-\mathrm{long}$ SPs. A CDP, synchronous with the leading edge of the contending Packet B, is generated for both the cases of Packet B delayed or advanced with respect to Packet A (cases of PCK A3-PCK B1 and PCK A1-PCK B3 contentions, respectively, in Fig 2c). No CDP is generated in absence of contention. The AOFF output signal and the corresponding 1x2 AOS_OFF/ON outputs are also shown. The HP packet sequence (Packets A) and 1x2 AOS_OFF output are recombined at OUT 1. The two last cases of interest, with more than two contending packets, are shown in Fig. 2d) and e). For the case of Fig. 2d), one SP A interacts with two SPs $B_{\mathrm{fs}}$ in the PMF, generating two BDGs. The probe pulse is partially reflected by the first grating, producing a CDP for the advanced Packet B; the partially transmitted probe is then reflected by the second grating and a second CDP, signaling the delayed contending Packet $\mathrm{B}$, is generated. Two switching signals are produced by the AOFF deflecting the two LP packets. For the case of Fig. 2e), two SPs A and one SP $B_{\mathrm{fs}}$ interact in the fiber; two probe pulses are reflected at different instants in different positions along the fiber. However, the two CDPs exit the DES at the same instant; indeed, they signal the same LP packet, and the switching signal at the AOFF output must then be the same. The superposition of these CDPs, albeit noisier than a single CDP, correctly triggers the AOFF. The BDG decay time constant of few ns secures that the gratings are only seen by the probe pulse immediately following SP A (with a delay of about $2.5 \mathrm{~ns}$ ). The average input power levels of SP A, SP $B_{\mathrm{fs}}$ and probe signal at the PMF were $+11,+10$, and $+8 \mathrm{dBm}$, respectively; input/output time traces at the DES are shown in Fig. 2b). Fig. 2f) shows the results of the BER for the input/output packets. A maximum power penalty of about $1.7 \mathrm{~dB}$ (at a BER $=10^{-9}$ ) is observed at the outputs.

In this demonstration, for sake of simplicity, priority is assigned by the input port; the scheme can be simply updated for assigning priority to a specific packet by using priority labels and inserting on the paths of both packets a $1 \times 2$ AOS, driven by an AOFF. By sending probe pulses from both sides of the SBS-ODL (thus making the scheme symmetric) priority can be decided by the presence or not of the probe pulse (acting as a priority label).

\section{Conclusions}

A continuously tunable variable ODL is exploited to demonstrate a novel concept for all-optical, bit-rate independent, contention resolution of asynchronous packets for optical packet switching networks, enabling operation on a priority basis. In this demonstration, priority is assigned to the input port. Extension for symmetric operation allowing priority swapping between the input packets by means of priority labels has been illustrated. Error-free packet CR without packet fragmentation is demonstrated.

This work has been carried out within the FP7, FET-Open, Project GOSPEL.

\section{References}

[1] S. Rangarajan, Z. Hu, L. Rau, D.J. Blumenthal, "All-Optical Contention Resolution With Wavelength Conversion for Asynchronous Variable-Length 40 Gb/s Optical Packets", Photon. Technol. Lett., 16, 689-691 (2004).

[2] D. Apostolopoulos et al., "Contention Resolution for Burst-Mode Traffic Using Integrated SOA-MZI Gate Arrays and Self-Resetting Optical Flip-Flops", Photon. Technol. Lett., 20, 2024-2027 (2008).

[3] N. Primerov, C. Sanghoon, K. Yong Song, L. Thévenaz, "Ultra wide range tunable delay line using dynamic grating reflectors in optical fibers", in Proc. OFC'10, OWF6 (2010)

[4] A. Trita et al, "10 Gb/s operation of Monolithic All-Optical Set-Reset Flip-Flop based on Semiconductor Ring Laser" in Proc. CLEO/QELS'10, CThN3 (2010). 